Hence our differential equation becomes

$$
\frac{c}{\sqrt{Y^{2}-c^{2}}}=\frac{Y^{2}}{Y^{\prime}},
$$

the integral of which is

$$
\frac{\sqrt{Y^{2}-c^{2}}}{Y c}=X+\text { constant. }
$$

If we substitute the old variables, this becomes

$$
\left(x^{2}+y^{2}\right)\left(k^{2} c^{2}-1\right)+2 c^{2} k x+c^{2}=0,
$$

where $k$ is an arbitrary constant, and this is the required general integral.

Again, we know that if $f(x, y, p)=0$ admits the group $\xi f_{x}+\eta f_{y}, f(x, y, \eta / \xi)=0$ satisfies the equation, and contains as factor any singular solution of the equation. If there are any other factors, they are particular cases of the general integral for certain values of the arbitrary constant.

In our case $f(x, y, \eta / \xi)=0$ becomes

$$
\left[\left(x^{2}+y^{2}\right)^{2}-c^{2} y^{2}\right]\left(x^{2}+y^{2}\right)=0 .
$$

Here $x^{2}+y^{2}=0$ is the general integral when $k=\infty$, and

$$
\left(x^{2}+y^{2}\right)^{2}-c^{2} y^{2}=0
$$

is the singular solution.

BRYN MAWr, PA., October, 1904.

\title{
ON THE QUINTIC SCROLL HAVING A TACNODAL OR OSCNODAL CONIC.
}

\author{
BY PROFESSOR VIRGIL SNYDER.
}

(Read before the American Mathematical Society, October 29, 1904.)

Besides the quintic scrolls having three double conics which were discussed in the BuLLETIN (volume 9, pages 236-242), other particular types exist. Two of the double conics may become consecutive, forming a tacnodal conic ; or all three may become consecutive, forming an oscnodal conic. The necessary 
condition that a nodal curve on a scroll be counted as two consecutive nodal curves is that the two generators which intersect at any point on the curve shall lie in a plane containing the tangent to the curve at that point.

1. Let $x=\lambda, y=\lambda^{2}, z=0, w=1$ be a conic $c_{1}$. The tangent plane at $\lambda$ and passing through the point $(0,0,1,1)$ is $\lambda^{2}(z-w)+2 \lambda x-y=0$. Consider a second conic $c_{2}, x=0$, $z^{2}-y w=0$. This conic cuts $c_{1}$ in two points, $(0,0,0,1)$, $(0,1,0,0)$ and its plane passes through the vertex $(0,0,1,1)$. The latter also lies in the common tangent plane of $c_{1}$ and $c_{2}$ at $(0,0,0,1)$. A tangent plane to $c_{1}$ at $\lambda$ and passing through $(0,0,1,1)$ will cut $c_{2}$ in two points $\left(0, y_{1}, z_{1}, w_{1}\right)$. If each such point on $c_{2}$ be joined with $\lambda$ by straight lines, a scroll will be defined having $c_{1}$ as tacnodal conic. If any point on $c_{2}$ be joined to $(0,0,1,1)$ and through the joining line the tangent planes be drawn to $c_{1}$, it is seen that $c_{2}$ is a double conic. Between $c_{1}, c_{2}$ exists therefore a $(2,2)$ correspondence having $(0,1,0,0)$ for a self-corresponding point and $(0,0,0,1)$ for double element. The scroll is consequently a unicursal quintic. On performing the algebraic work indicated, the equation is found to be

$$
\left(y w-z^{2}-x^{2}\right)^{2}(z-w)+2 x^{2} z\left(y w-z^{2}-x^{2}\right)-y z^{2} x^{2}=0 .
$$

The simple conic of the surface * lies in the plane $z-w=0$ and touches $c_{1}$ at $(0,1,0,0)$. An arbitrary plane will cut the scroll in a quintic curve having two double points and two tacnodes. A plane containing a tangent to $c_{1}$ will contain a quintic having four consecutives nodes. The common tangent plane to $c_{1}$ and $c_{2}$ at $(0,0,0,1)$ contains a nodal cubic and two generators for nodal tangents. by

2. Now let $c_{1}, c_{2}$ touch each other. They may be defined

$$
\begin{aligned}
& c_{1}: \quad z=0, \quad y^{2}-x w=0 ; \\
& c_{2}: \quad x=0, \quad y^{2}-w z=0 .
\end{aligned}
$$

In parametric form $c_{1}$ becomes

$$
x=\mu^{2}, \quad y=\mu, \quad z=0, \quad w=1 .
$$

\footnotetext{
* See Sisam, Bulletin, volume 10, page 32.
} 
The equations of a line joining $\mu$ on $c_{1}$ to $\left(0, y_{1}, z_{1}, w_{1}\right)$ on $c_{2}$ may be written

from which

$$
\frac{x-\mu^{2} w}{-\mu^{2} w_{1}}=\frac{y-\mu w}{y_{1}-\mu w_{1}}=\frac{z}{z_{1}}
$$

$$
\frac{\mu^{2} w-x}{\mu^{2} z}=\frac{w_{1}}{z_{1}}, \quad \frac{\mu y-x}{\mu z}=\frac{y_{1}}{z_{1}} \text {. }
$$

Since $\left(0, y_{1}, z_{1}, w_{1}\right)$ lies on $c_{2}$, we have

$$
\mu^{2}\left(y^{2}-w z\right)-\mu \cdot 2 x y+x^{2}+x z=0 .
$$

The equation of a tangent plane to $c_{1}$ at $\mu$ has the form

$$
\mu^{2} w-2 \mu y-\kappa z+x=0 .
$$

For a general value of $\kappa$, the surface is a sextic scroll, having $x=0, z=0$ as double generator. If the vertex of the cone lies on $c_{2}$, the latter is simple. If $\kappa=2 \mu-1$, the plane $x+z=0$ is a factor and $c_{2}$ is a double conic on the resulting $S_{5} . \quad c_{1}$ is a tacnodal conic.

3. A further specialization can occur, in which all three conics become consecutive. The possibility of this form was pointed out by Wiman,* who incidentally mentions it while describing scrolls having double conics, but does not discuss it nor derive its equation. Wiman employs the Noether point depiction of the lines of a linear complex into the points of space, projectively equivalent to the system which Lie investigated. The exceptional cases and fundamental configurations are treated in detail in my Göttingen dissertation. $\nmid$

Let

$$
x_{1} x+y_{1} y+w_{1} w=0, \quad y_{1} x+z_{1} y+w_{1} z=0 .
$$

define a relation between the points of one space and the lines of another. To the points $\left(x_{1}, y_{1}, z_{1}, w_{1}\right)$, or briefly $\left(x_{1}\right)$, correspond the lines of the complex

$$
c^{1}: \quad p_{14}+p_{23}=0,
$$

* Klassifikation af regelytorna af sjette graden, Lund (dissertation), 1892 ; page 87.

† Ueber-die linearen Complexe der Lie'schen Kugelgeometrie. 
and to the points of space $(x)$ correspond the lines of the quadratic complex

$c^{2}:$

$$
p_{34} p_{14}-p_{42}^{2}=0,
$$

which consists of all the lines which intersect the conic $w_{1}=0$, $y_{1}^{2}=x_{1} z_{1}$.

To a ruled surface belonging to $e^{1}$ corresponds a curve in $\left(x_{1}\right)$; to its double curve corresponds a ruled surface of bisecants, all of whose generators belong to $c^{2}$. The line $x=0, y=0$ is the fundamental line of $(x)$ and the plane $w_{1}=0$ is fundamental in $\left(x_{1}\right)$. To all points in $w_{1}=0$ corresponds the same line $x=0, y=0$. An important relation exists between the arrangement of points in $(x)$ and the lines of $c^{2}$ in $\left(x_{1}\right)$. If a point $\left(x_{1}, y_{1}, z_{1}\right)$ be given on $y_{1}^{2}-x_{1} z_{1}=0, w_{1}=0$, then a plane $x_{1} x+y_{1} y=0$ is defined in $(x)$. All the lines of the pencil belonging to $c^{1}$ and lying in the plane $x_{1} x+y_{1} y=0$ go over into the same point of the conic. All the points of one of these lines go over into lines of a pencil whose vertex is on the conic and which contains the tangent to the conic; hence all the points in the plane correspond to the pencils of lines of $c^{2}$ lying in the pencil of planes through a common tangent to the conic.

Given then a scroll $S_{m}$ of order $m$, in $\left(x_{1}\right)$, having the fundamental conic for a $\rho$-fold curve, in its plane lie $m-2 \rho$ generators ; hence to $S_{m}$ corresponds a curve $c_{m-\rho}$ of order $m-\rho$ in $(x)$ having $m-2 \rho$ points on the fundamental line $x=0, y=0$. To apply this depiction to an $S_{5}$ having three double conics, regard one of the conics as the fundamental conic of $c^{2}$ and transform the surface into point space. The generators will go into the points of a twisted cubic, cutting $x=0, y=0$ once, and the residual double conic will become two hyperboloids, each having two generators belonging to $c^{1}$ in its second system. If one of these lines be chosen as $x=0, y=0$ and the system be depicted back into $\left(x_{1}\right)$, the double conic will coincide with the fundamental conic counting as the tacnodal conic on $S_{5}$. This surface is then equivalent to that given before. If now the ordinary double conic be taken as fundamental conic, the surface transformed into points of $(x)$, and the same transformation of the fundamental line made as in the preceding case, then the $S_{5}$ which is the transform of the last system will have the fundamental conic as oscnodal curve.

From the equations of a generator we derive $y-\lambda x=z \zeta$, 
$\zeta(\lambda w-x)=\lambda x, \lambda^{2}(\zeta-1)=\zeta^{2}$. By letting $\lambda=\left(l^{2}+1\right) / l$, $\zeta=l^{2}+1$, the equations of a generator may be written

$$
\begin{aligned}
& l^{2} z_{1}-l w_{1}+z_{1}-y_{1}=0, \\
& l^{3} y_{1}+l^{2} w_{1}+l\left(y_{1}-x_{1}\right)+w_{1}=0 .
\end{aligned}
$$

Making these equations identical with (1), we obtain

$$
\sigma x=-l, \quad \sigma y=l\left(l^{2}+1\right), \quad \sigma z=-l^{2}, \quad \sigma w=l^{2}+1,
$$

as the equations of $c_{3}$, the $(x)$ image of the $S_{5}$. The curve $c_{3}$ lies on the hyperboloid $z w-x y=0$, which is to be transformed so that $x=0, y=0$ lies on the surface and cuts $c_{3}$ once, all the generators cutting $x=0, y=0$ shall belong to $c^{1}$, but the points of the surface shall not go over into lines of a special linear complex in $\left(x_{1}\right)$. The most general transformation of this type may be defined by

$$
y=y^{\prime}, \quad x=c y^{\prime}-d z^{\prime}, \quad z=x^{\prime}, \quad w=a x^{\prime}+b y^{\prime}+d w^{\prime},
$$

from which

$$
\begin{gathered}
x^{\prime}=-l^{2}, \quad y^{\prime}=l\left(l^{2}+1\right), \quad z^{\prime}=a l\left(l^{2}+1\right)+b l, \\
w^{\prime}=b\left(l^{2}+1\right)+c l^{2}+d l\left(l^{2}+1\right) .
\end{gathered}
$$

The values are to be substituted in (1) for $x, y, z, w$. The $l$ eliminant in $\left(x_{1}\right)$ has the form

$$
\left|\begin{array}{ccccc}
y+d w & c w-x & y+d w & b w & 0 \\
0 & y+d w & c w-x & y+d w & b w \\
z+a w & -y & z+c w & 0 & 0 \\
0 & z+a w & -y & z+c w & 0 \\
0 & 0 & z+a w & -y & z+c w
\end{array}\right|=0
$$

after a few obvious changes are made in the constants.

A plane through any tangent to the oscnodal conic will cut from the surface a quintic having six consecutive double points. A plane containing two generators contains a nodal cubic having the generators as nodal tangents.

CORNELL UNIVERSTTY, October 18, 1904 . 